TRANSACTIONS OF THE

AMERICAN MATHEMATICAL SOCIETY

Volume 196, 1974

\title{
ANALYTIC EQUIVALENCE \\ AMONG SIMPLY CONNECTED DOMAINS IN $C(X)$
}

BY

\author{
HUGH E. WARREN
}

\begin{abstract}
This work considers analytic equivalence within the analytic function theory for commutative Banach algebras which was introduced by $E$. $R$. Lorch. Necessary conditions of a geometric nature are given for simply connected domains in $C(X)$. These show that there are a great many equivalence classes. In some important cases, as when one domain is the unit ball, the given conditions are also sufficient. The main technique is the association of a simply connected domain in $C(X)$ with a family of Riemann surfaces over the plane.
\end{abstract}

1. Introduction. There are many extensions of analytic function theory to settings other than one complex variable. In any such extension one can raise the question of when two sets are analytically equivalent, that is, of when two sets support isomorphic families of analytic functions. In this paper the underlying analytic function theory is that due to E. R. Lorch [7]. The space in which analytic functions will be defined and take their values is $C(X)$. This space is the commutative Banach algebra of continuous complex valued functions on a compact Hausdorff space $X$. The norm in $C(X)$ is the uniform norm. An analytic function in $C(X)$ is one that can be locally represented by power series $f \rightarrow \Sigma g_{n}(f-g)^{n}$ where $g$ and $g_{n}$ belong to $C(X)$ and the series converges in the norm of $C(X)$ on a ball about $g$.

The problem of characterizing the sets in $C(X)$ which are analytically equivalent to the unit ball was previously considered by the author in [9] and [10]. The present paper develops methods which solve that problem and which say a good deal about the equivalence problem for simply connected domains in general. Necessary conditions for equivalence are given which show that the number of equivalence classes must be very large.

The principal analytic device is the association of each simply connected domain in $C(X)$ with a collection of Riemann surfaces which are called

Presented to the Society, January 25, 1973; received by the editors February 7, 1973. AMS (MOS) subject classifications (1970). Primary 30A96, 46J10.

Key words and phrases. Lorch analytic function, analytic equivalence, Riemann surface. 
the sections of the domain. An analytic equivalence between two domains induces analytic equivalences between their sections. In appropriate circumstances equivalences between sections can be assembled into an equivalence between the domains.

Beyond analytic equivalence the sections of a simply connected domain lead to an extension of the notion of taking quotient functions. Quotient functions have been important in the Lorch function theory. For a description see [2].

A good deal of specialized notation is necessary. To begin, the uniform norm of a function $f \in C(X)$ is $\|f\|=\sup \{|f(x)| ; x \in X\}$. For $\epsilon>0$ the open ball of radius $\epsilon$ about $f$ is $B(f, \epsilon)=\{g \in C(X) ;\|g-f\|<\epsilon\}$. Write $C$ for the complex numbers and $D(z, \epsilon)$ for the disk of radius $\epsilon$ about $z$. We embed $C$ in $C(X)$ by identifying each complex number with the constant function on $X$ which takes that complex value. If $f \in C(X)$ and $z \in C$, then $f+z$ is the function whose value at $x \in X$ is $f(x)+z$. Write $B$ for $B(0,1)$ and $D$ for $D(0,1)$. The set $B$ is the open unit ball of $C(X)$.

An open connected set will be called a domain. We shall speak of domains in $C$ and in $C(X)$, according to context. The letters $U$ and $V$ will be reserved for domains in $C(X)$. Domains $U$ and $V$ are (analytically) equivalent if there exists a one-to-one analytic map $\Phi$ of $U$ onto $V$ whose inverse is also analytic. We shall refer to $\Phi$ as an (analytic) equivalence of $U$ onto $V$. An equivalence $\Phi$ has the property that the derivative $\Phi^{\prime}[f]$ at any function $f \in U$ is a nowhere vanishing function. If conversely $\Phi^{\prime}[f]$ is nowhere vanishing, then $\Phi$ is an equivalence on some neighborhood of $f$. See the introductory remarks in [2]. If $g, b \in C(X)$ and $b$ is nowhere vanishing, then the maps $f \rightarrow f+g$ and $f \rightarrow b f$ are equivalences of $C(X)$ onto itself. Thus it is no restriction of the equivalence problem to make assumptions such as $0 \in U$ and $\Phi^{\prime}[0]=1$.

Given a function $f$ in a domain $V$ we write $B_{V}(f)$ for the largest ball $B(f, \epsilon)$ contained in $V$. When context is unambiguous, write simply $B(f)$. For each $x \in X$ we have the evaluation map $E_{x}: C(X) \rightarrow C$ defined by $E_{x}(f)=f(x)$. Given $f \in V$ put $V_{x}=E_{x}(V)$ and $D[x, f]=E_{x}(B(f))$. The dependence of $D[x, f]$ on $V$ will be left implicit. Note that $D[x, f]$ is a disk in $V_{x}$ centered at $f(x)$.

The letters $\Phi$ and $\Psi$ will be used for mappings from $C(X)$ into $C(X)$. The value of $\Phi$ at $f \in C(X)$ is the function $\Phi[f]$, and the value of $\Phi[f]$ at $x \in X$ is $\Phi[f](x)$. Juxtaposition will denote composition, in the manner of operators. For example $\Psi \Phi[f]=\Psi[\Phi[f]]$ and $E_{x} \Phi[f]=\Phi[f](x)$. However, $f g$ is the usual product function when $f, g \in C(X)$.

The letters $\gamma$ and $\eta$ will denote paths in various spaces. The same symbol will be used both for the function that defines the path and for the image set. The sum $\gamma+\eta$ has the usual meaning: traverse $\gamma$, then traverse $\eta$. For $w, z \in C$ 
write $[w, z]$ for the straight line path on $C$ from $w$ to $z$. For $f, g \in C(X)$ write $[f, g]$ for the straight line path in $C(X)$ from $f$ to $g$.

Two Riemann surfaces are called equivalent if there is a bijection between them which is holomorphic (in both directions). The bijection will be referred to as an equivalence between the two surfaces. We shall rely heavily on the uniformization theorem [1], that a simply connected, noncompact Riemann surface is equivalent either to the plane or to the disk.

2. Sections. In the discussion under this heading $V$ will be a simply connected domain in $C(X)$ and $x$ will be a point of $X$. For each $f \in V$ let $\Delta(x, f)$ be a copy of the planar disk $D[x, f]$. Think of $\Delta(x, f)$ as lying above $D[x, f]$. Let $P$ be the projection of $\Delta(x, f)$ onto $D[x, f]$. Let $M_{x}$ be the disjoint union of the disks $\Delta(x, f), f \in V$. Define $\mu_{x}: V \rightarrow M_{x}$ by $\mu_{x}(f) \in \Delta(x, f)$ and $P \mu_{x}(f)=$ $f(x)$. In other words $\mu_{x}(f)$ is the center point of $\Delta(x, f)$.

Let $\Omega$ be the first uncountable ordinal. For the ordinal numbers $j=0,1, \ldots, \Omega$ we shall inductively define equivalence relations $R_{j}$ on $M_{x}$. We write $S_{x, j}$ for the quotient space $M_{x} / R_{j}$. Our goal is to define $R_{\Omega}$ so that $S_{x, \Omega}$ is a simply connected Riemann surface. We shall call $S_{x, \Omega}$ the section of $V$ at $x$.

Define $w R_{0} z$ to mean $P w=P z$. Suppose $R_{j}$ has been defined for $j<k$. Let $Q_{j}: M_{x} \rightarrow S_{x, j}$ be the quotient map, and put $\Delta[x, f]_{j}=Q_{j}(\Delta(x, f))$. Define $\sigma_{x, j}: V \rightarrow S_{x, j}$ by $\sigma_{x, j}=Q_{j} \mu_{x^{*}}$. Define $P: S_{x, j} \rightarrow C$ by $P Q_{j} w=P w, w \in M_{x^{*}}$ As part of the induction hypothesis we assume that for $j<k$

(1) $w R_{j} z$ and $i<j$ imply $w R_{i} z$;

(2) if $\Delta[x, f]_{j} \cap \Delta[x, g]_{j}$ is not empty and if $w \in \Delta(x, f), z \in \Delta(x, g)$ with $P w=P z$, then $Q_{j} w=Q_{j} z$;

(3) $Q_{j}$ is an embedding of $\Delta(x, f)$ and $\Delta[x, f]_{j}$ is open in $S_{x, j}$;

(4) $\sigma_{x, j}$ is continuous.

Condition (1) with $i=0$ and condition (2) say that the action of $Q_{j}$ is to identify certain disks in $M_{\boldsymbol{x}}$ where their projections overlap. Condition (3) says that $S_{x, j}$ is a 2-manifold and that the disks $\Delta[x, f]_{j}$ are coordinate patches. Condition (4) insures that if $\gamma$ is a path in $V$, then the composition $\sigma_{x, j} \gamma$ is a path in $S_{x, j}$.

The map $P$ projects $\Delta[x, f]_{j}$ one-to-one onto $D[x, f]$. This is because $P Q_{j}=P$ and because $P$ projects $\Delta(x, f)$ one-to-one onto $D[x, f]$. In view of (2) if $\Delta[x, f]_{j}$ and $\Delta[x, g]_{j}$ have nonempty intersection, then $P$ projects the union of these two disks one-to-one onto $D[x, f] \cup D[x, g]$. So when $\Delta[x, f]$ and $\Delta[x, g]_{j}$ overlap it makes sense to speak of the straight line path in $S_{x, j}$ from $\sigma_{x, j}(f)$ to $\sigma_{x_{0} j}(g)$. The symbol $[f, g]_{x, j}$ will denote this path.

We define $w R_{k} z$ to mean that for $j<k$ 
(1') $w R_{j} z$ and

$\left(2^{\prime}\right)$ if $w \in \Delta(x, f)$ and $z \in \Delta(x, g)$ and if $\gamma$ is a path in $V$ from $f$ to $g$, then $\sigma_{x, j} \gamma$ is homotopic in $S_{x, j}$ to $[f, g]_{x, j}$.

In $\left(2^{\prime}\right)$ note that $[f, g]_{x, j}$ exists because $Q_{j} w=Q_{j} z$ is a point common to $\Delta[x, f]_{j}$ and $\Delta[x, g]_{j}$. The paths $\sigma_{x, j} \gamma$ and $[f, g]_{x, j}$ have the same initial and final points, namely $\sigma_{x, j}(f)$ and $\sigma_{x, j}(g)$.

To justify this inductive definition we first verify (1) through (4) when $j=0$. Condition (1) is vacuously true. Since $w R_{0} z$ means $P w=P z$, the map $Q_{0}$ makes the same identifications as $P$. We can thus identify $Q_{0}$ with $P$ and $S_{x, 0}$ as a set with $P\left(M_{x}\right)$. We have

$$
P\left(M_{x}\right)=\bigcup_{f \in V} P(\Delta(x, f))=\bigcup_{f \in V} D[x, f]=\bigcup_{f \in V} E_{x}(B(f))=E_{x}(V)=V_{x^{*}}
$$

Since $P$ is an equivalence of $\Delta(x, f)$ onto $D[x, f]$, the quotient topology of $S_{x, 0}$ matches the relative topology of $V_{x}$ in the plane. Thus $S_{x_{0}, 0}$ coincides with the starting section $V_{x}$. Conditions (2) and (3) follow from $Q_{0}=P$, and (4) follows from $\sigma_{x, 0}=E_{x}$.

We next show that (1) through (4) are satisfied when $j=k$. Condition (1) is the same as $\left(1^{\prime}\right)$. To show (2) suppose $w, w^{\prime} \in \Delta(x, f)$ and $z, z^{\prime} \in \Delta(x, g)$ with $Q_{k} w^{\prime}=Q_{k} z^{\prime}$ and $P w=P z$. Since $w^{\prime} R_{k} z^{\prime}$, condition (2') holds for $j<k$. From $w R_{0} z$, which is the same as $P w=P z$, and from (2') with $j=0$ we get $w R_{1} z$. By adding ( $\left.2^{\prime}\right)$ with $j=1$ we get $w R_{2} z$, and so on. By induction $w R_{k} z$.

Before checking (3) we verify that $R_{k}$ is an equivalence relation on $M_{x}$. Let $w, z, \zeta$ be points of $M_{x}$ with $w \in \Delta(x, f), z \in \Delta(x, g), \zeta \in \Delta(x, b)$. The subscript $j$ is understood to vary over $0 \leq j<k$. Condition (1') meets the conditions of reflexivity, symmetry, and transitivity because $R_{j}$ is an equivalence relation. We proceed to check $\left(2^{\prime}\right)$ against these requirements. Let $\gamma$ be a path in $V$ from $f$ to $f$. Since $V$ is simply connected, $\gamma$ is homotopic in $V$ to the point path $[f, f]$. Hence $\sigma_{x, j} \gamma$ is homotopic in $S_{x, j}$ to $[f, f]_{x, j}$. This shows that $w R_{k} w$, so $R_{k}$ is reflexive. Suppose that $w R_{k} z$ and that $\eta$ is a path in $V$ from $g$ to $f$. Since $-\eta$ goes from $f$ to $g$, the relation $w R_{k} z$ implies $-\sigma_{x, j} \eta$ is homotopic to $[f, g]_{x, j}$. Thus $\sigma_{x, j} \eta$ is homotopic to $[g, f]_{x, j}$ and $z R_{k} w$. This establishes that $R_{k}$ is symmetric.

Suppose that $w R_{k} z$ and $z R_{k} \zeta$. Let $\gamma$ be a path in $V$ from $f$ to $g$ and $\eta$ a path in $V$ from $f$ to $b$. The path $-\gamma+\eta$ goes from $g$ to $b$. The relation $w R_{k} z$ implies $\sigma_{x, j} \gamma$ is homotopic to $[f, g]_{x, j}$, and $z R_{k} \zeta$ implies $-\sigma_{x, j} \gamma+\sigma_{x, j} \eta$ is homotopic to $[g, b]_{x, j}$. Thus $\sigma_{x, j} \eta$ is homotopic to $[f, g]_{x, j}+[g, b]_{x, j}$. Since $R_{j}$ is transitive, $Q_{j} w=Q_{j} z=Q_{j} \zeta$ is a point in the intersection of $\Delta[x, f]_{j}, \Delta[x, g]_{j}$, , 
and $\Delta\left[x_{0}, b\right]_{j}$. The union of these same disks is equivalent under $P$ to $D[x, f] \cup D[x, g] \cup D[x, b]$. In the latter union $[f(x), g(x)]+[g(x), b(x)]$ is homotopic to $[f(x), b(x)]$. It follows that $[f, g]_{x, j}+[g, b]_{x, j}$ and hence $\sigma_{x, j} \eta$ are homotopic in $S_{x, j}$ to $[f, b]_{x, j}$. Thus $w R_{k} \zeta$, and $R_{k}$ is transitive.

Now check condition (3). By (1') the equality $Q_{k} w=Q_{k} z$ implies $P w=P z$. The map $P$ is one-to-one on $\Delta(x, f)$, and $P Q_{k}=P$; therefore $Q_{k}$ is one-to-one on $\Delta(x, f)$. In the quotient topology of $S_{x, k}$ a neighborhood base at a point $Q_{k} w$ consists of sets of the form $\bigcup_{Q_{k}}\left(N_{g}\right)$ where the union is over $g \in V$ and $N_{g}$ is either a neighborhood in $\Delta(x, g)$ of a point $z$ with $w R_{k} z$ or empty if no such $z$ exists. Say $w \in \Delta(x, f)$, and let $N$ be a neighborhood of $w$ in $\Delta(x, f)$. For each $g \in V$ put $N_{g}=\varnothing$ if $Q_{k} w \notin \Delta[x, g]_{k}$ and otherwise let $N_{g} \subset \Delta(x, g)$ be determined by $P\left(N_{g}^{g}\right)=P(N) \cap D[x, g]$. With this choice of the $N_{g}^{g}$ we have $Q_{k}(N)=$ $\cup Q_{k}\left(N_{g}\right)$. This means $Q_{k}(N)$ is a neighborhood in $S_{x, k}$ of $Q_{k} w$. Letting $w$ and $N$ vary over all possibilities shows (3) is true.

The next result leads up to condition (4) but is important in its own right.

Lemma 2.1. If $g \in B(f)$, then $\sigma_{x, k}(g) \in \Delta[x, f]_{k}$.

Proof. From $g \in B(f)$ we get $g(x) \in E_{x}(B(f))=D[x, f]$. The equation $P w=$ $g(x)$ thus determines a point $w \in \Delta(x, f)$. The relation $\mu_{x}(g) R_{0} w$ follows from $P w=g(x)=P \mu_{x}(g)$. In the simply connected domain $V$ any path $\gamma$ from $g$ to $f$ is homotopic to $[g, f]$, which lies in $V$ because $g \in B(f)$. Consequently $\sigma_{x, j} \gamma$ is homotopic in $S_{x, j}$ to $[g, f]_{x, j}=\sigma_{x, j}[g, f]$ when $j<k$, that is, $\left(2^{\prime}\right)$ holds for $0 \leq j<k_{0}$ From this fact and from $\mu_{x}(g) R_{0} w$ an induction gives $\mu_{x}(g) R_{k} w$. To finish the proof,

$$
\sigma_{x, k}(g)=Q_{k} \mu_{x}(g)=Q_{k} w \in \Delta[x, f]_{k} .
$$

Turning to condition (4), define neighborhoods $N_{\epsilon} \subset \Delta(x, n)$ by $P\left(N_{\epsilon}\right)=$ $D(f(x), \epsilon)$. By $(3)$ the sets $Q_{k}\left(N_{\epsilon}\right)$ are a neighborhood base at $\sigma_{x, k}(f)$. If $g \in B(f, \epsilon) \subset B(f)$, then $\sigma_{x, k}(g) \in Q_{k}\left(N_{\epsilon}\right)$. This means that $\sigma_{x, k}$ is continuous.

Transfinite induction defines $S_{x_{, j}}$ for $0 \leq j \leq \Omega$. In addition (1) through (4) are satisfied.

Lemma 2.2. Every point of $S_{x, j}$ bas the form $\sigma_{x, j}(f)$ for some $f \in V$.

Proof. For $w \in \Delta[x, g]_{j}$ put $f=g+(P w-g(x))$. This gives $f \in B(g)$ and $w=\sigma_{x, j}(f)$.

Lemma 2.3. Each $S_{x, j}$ is patb connected.

Proof. Given $w=\sigma_{x, j}(f)$ and $z=\sigma_{x, j}(g)$, let $\gamma$ be a path in $V$ from $f$ to $g$. The path $\sigma_{x, j} \gamma$ connects $w$ to $z$. 
Lemma 2.4. Each $S_{x, j}$ is a Riemann surface.

Proof. The projection $P$ is an equivalence of $\Delta[x, f]$ onto $D[x, f]$, and $\left\{\Delta[x, f]_{j} ; f \in V\right\}$ is an open cover of $S_{x, j}$. By (2) the complex structures of $\Delta[x, f]_{j}$ and $\Delta[x, g]_{j}$ match up if the two disks overlap. Since $S_{x, j}$ is connected, it is a Riemann surface.

Lemma 2.5. There exists an ordinal number $J<\Omega$ with the property that $k \geq J$ implies $S_{x, k}=S_{x, J}$.

Proof. The Riemann surface $S_{x, \Omega}$ is separable. From the open cover $\left\{\Delta[x, f]_{\Omega} ; f \in V\right\}$ extract a countable subcover $\left\{\Delta\left[x, f_{n}\right]_{\boldsymbol{\Omega}} ; n=1,2\right.$, etc. $\}$. For pairs of positive integers $(n, m)$ define $j(n, m)$ as follows. If $\Delta\left[x, f_{n}\right]_{\Omega}$ and $\Delta\left[x, f_{m}\right]_{\Omega}$ have nonempty intersection, put $j(n, m)=0$. If $\Delta\left[x, f_{n}\right]_{\Omega}$ and $\Delta\left[x, f_{m}\right]_{\Omega}$ are disjoint let $j(n, m)$ be the least ordinal $j$ with the property that $\Delta\left[x, f_{n}\right]_{j}$ and $\Delta\left[x, f_{m}\right]_{j}$ are disjoint. We show that $j(n, m)<\Omega$. Suppose $\Delta\left[x, f_{n}\right]_{j}$ and $\Delta\left[x, f_{m}\right]_{j}$ have nonempty intersection for all $j<\Omega$. In particular we have $w \in \Delta\left(x, f_{n}\right)$ and $z \in \Delta\left(x, f_{m}\right)$ with $Q_{0} w=Q_{0} z$, which is equivalent to $P w=P z$. For all $j<\Omega$

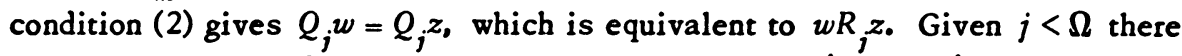
is a $k$ with $j<k<\Omega$. The relation $w R_{k} z$ holds, so $\left(1^{\prime}\right)$ and $\left(2^{\prime}\right)$ hold. Since $j$ is any ordinal less than $\Omega$, we have $w R_{\Omega} z$ by definition. Thus $\Delta\left[x, f_{n}\right]_{\Omega}$ and $\Delta\left[x, f_{m}\right]_{\Omega}$ both contain $Q_{\Omega} w=Q_{\Omega} z$, and $j(n, m)=0$.

The supremum of the countably many ordinals $j(n, m)<\Omega$ is some ordinal $J<\Omega$. This is the $J$ in the statement of the lemma. For suppose $w \in \Delta\left(x, f_{n}\right)$ and $z \in \Delta\left(x, f_{m}\right)$ with $Q_{J} w=Q_{J} z$. In particular $\Delta\left[x, f_{n}\right]$, and $\Delta\left[x, f_{m}\right]$, have nonempty intersection. Since $J \geq j(n, m)$, it must be that $\Delta\left[x, f_{n}\right]_{\Omega}$ and $\Delta\left[x, f_{m}\right]_{\Omega}$ have nonempty intersection. The relation $Q_{J} w=Q_{J} z$ implies $P w=P z$, and condition (2) with $j=\Omega$ then implies $Q_{\Omega} w=Q_{\Omega} z$. That is, $w R_{J} z$ implies $w R_{\Omega} z$.

Recall that $\left\{\Delta\left[x, f_{n}\right]_{\Omega^{\prime}} n=1,2\right.$, etc. $\}$ covers $S_{x, \Omega^{*}}$. For any $w^{\prime}, z^{\prime} \in M_{x}$ there are $w$ and $z$ as above with $w R_{\Omega} w^{\prime}$ and $z R_{\Omega} z^{\prime}$, hence $w R_{J} w^{\prime}$ and $z R_{J}^{x} z^{\prime}$. If $w^{\prime} R_{J} z^{\prime}$, then $w R_{J} z$ because $R_{J}$ is an equivalence relation. Consequently $w R_{\Omega} z$, and since $R_{\mathbb{Q}}$ is an equivalence relation, $w^{\prime} R_{\Omega} z^{\prime}$. This means $R_{J}$ is at least as strong as $R_{\mathbb{Q}}$. From condition (1) we deduce that $R_{k}=R_{J}$ whenever $J \leq k<\Omega$. Since $S_{x, k}=M_{x} / R_{k}$, we are done.

Theorem 2.6. The surface $S_{x, Q}$ is simply connected.

Proof. Given a closed path $\gamma$ in $S_{x, \&}$ consider $\gamma$ to be a continuous mapping of $[0,1]$ into $S_{x, \Omega}$ with $\gamma(0)=\gamma(1)$. Since the range of $\gamma$ is compact, it is covered by finitely many disks $\Delta\left[x_{,} g_{i}\right]_{Q^{*}}$ As $t$ increases from 0 to 1 the image $\gamma(t)$ successively enters and leaves these disks. Renumber the $g_{i}$, with repetitions 
and omissions as necessary, and partition $[0,1]$ into successive intervals $\left[t_{i-1}, t_{i}\right]$ so that $\gamma(t) \in \Delta\left[x, g_{i}\right]_{\mathbb{a}}$ for $t_{i-1} \leq t \leq t_{i}$ and $i=1, \ldots, n$. We agree that $t_{0}=0$ and $t_{1}=1$. We put $g_{0}=g_{n}$.

For $1 \leq i \leq n$ we have $\gamma\left(t_{i-1}\right) \in \Delta\left[x, g_{i-1}\right]_{\Omega} \cap \Delta\left[x, g_{i}\right]_{\Omega}$. Condition (2) with $j=\Omega$ shows that the union of $\Delta\left[x, g_{i-1}\right]_{\Omega}$ and $\Delta\left[x, g_{i}\right]_{\Omega}$ contains the straight line path $\left[g_{i-1}, g_{i}\right]_{x, Q^{\circ}}$ Put

$$
\eta=\left[g_{0}, g_{1}\right]_{x, \Omega}+\cdots+\left[g_{n-1}, g_{n}\right]_{x, \Omega}
$$

The paths $\gamma$ and $\eta$ are homotopic in $\bigcup_{i} \Delta\left[x, g_{i}\right]_{\Omega}$, hence homotopic in $S_{x, \Omega^{*}}$. To prove the theorem it suffices to show $\eta$ is nullhomotopic in $S_{x, \Omega^{\circ}}$

Choose $w_{i} \in \Delta\left(x, g_{i-1}\right)$ and $z_{i} \in \Delta\left(x, g_{i}\right)$ with $Q_{\Omega} w_{i}=Q_{\Omega} z_{i}=\gamma\left(t_{i-1}\right)$. Let $\eta_{i}$ be a path in $V$ from $g_{i-1}$ to $g_{i}$. Let $J$ be the ordinal number of Lemma 2.5. Since $w_{i} R_{\Omega} z_{i}$ and $J<\Omega$, condition (2') with $j=J$ shows that $\sigma_{x, J} \eta_{i}$ is homotopic in $S_{x, J}$ to $\left[g_{i-1}, g_{i}\right]_{x, J}$. By Lemma $2.5, R_{J}=R_{Q}$, and so $\sigma_{i, \Omega} \eta_{i}$ is homotopic in $S_{x, \Omega}$ to $\left[g_{i-1}, g_{i}\right]_{x, \Omega}$. Summing these paths for $1 \leq i \leq n$, we see that $\sigma_{x, \Omega}\left(\eta_{1}+\cdots+\eta_{n}\right)$ is homotopic to $\eta$. The path $\eta_{1}+\cdots+\eta_{n}$ is closed, hence nullhomotopic in $V$. In turn $\sigma_{x, \Omega}\left(\eta_{1}+\cdots+\eta_{n}\right), \eta$ and $\gamma$ are nullhomotopic in $S_{x, \Omega^{\circ}}$

In place of $S_{x, \Omega}, \Delta[x, f]_{\Omega}$, etc., write $s_{x}, \Delta[x, f]$, etc. That is, from now on suppress the subscript $\Omega$. To emphasize the role of $V$ we shall frequently write $S_{x}(V)$ for $S_{x}$. For examples of domains $V$ see the last part of [10] and the end of this paper.

3. Sections and equivalence. Here we investigate the relation between sections of a simply connected domain $V$ and analytic functions defined on $V$. Let $\Phi: V \rightarrow C(X)$ be analytic. Fix a point $x \in X$ and define $\Phi_{x}: S_{x}(V) \rightarrow C$ as follows. For each $g \in V$ let $\Phi[f]=\Sigma g_{n}(f-g)^{n}$ be the power series expansion of $\Phi$ at $g$. Write the points of the disk $\Delta[x, g] \subset S_{x}(V)$ in the form $\sigma_{x}(g)+z$, where $z$ is a complex number and $P\left(\sigma_{x}(g)+z\right)=g(x)+z$. Define $\Phi_{x}$ on $\Delta[x, g]$ by

$$
\Phi_{x}\left(\sigma_{x}(g)+z\right)=\sum_{n=0}^{\infty} g_{n}(x) z^{n} \text {. }
$$

The collection $\{\Delta[x, g] ; g \in V\}$ covers $S_{x}(V)$; therefore $\Phi_{x}$ is defined on all of $S_{x}(V)$, although possibly many valued.

Lemma 3.1. The mapping $\Phi_{x}$ is bolomorphic on $S_{x}(V)$.

Proof. Any function element given by (3.1) is an analytic continuation of any other. To see this let $g$ and $b$ be any two functions in $V$. Choose functions $g=f_{0}, f_{1}, \cdots, f_{n}=b$ in $V$ so that $f_{i} \in B\left(f_{i-1}\right)$. This is possible because $V$ is 
a domain. Since $\Phi$ is analytic, the power series expansions of $\Phi$ at $f_{i-1}$ and $f_{i}$ are rearrangments of each other. Consequently the power series expansion of $\Phi_{x}$ at $\sigma_{x}\left(f_{i}\right)$ is a rearrangement of the expansion at $\sigma_{x}\left(f_{i-1}\right)$. Lemma 2.1 shows that $\sigma_{x}\left(f_{i}\right) \in \Delta\left[x, f_{i-1}\right]$; therefore the function element of $\Phi_{x}$ at $\sigma_{x}(b)=\sigma_{x}\left(f_{n}\right)$ is a continuation of the element at $\sigma_{x}(g)=\sigma_{x}\left(f_{0}\right)$.

Theorem 2.6 shows that $S_{x}(V)$ is simply connected. The monodromy theorem thus implies that $\Phi_{x}$ is single valued.

We call $\Phi_{x}$ the quotient function of $\Phi$ at the point $x$. Putting $z=0$ in (3.1) we get the characteristic relation

$$
\Phi_{x}\left(\sigma_{x}(g)\right)=\Phi[g](x),
$$

or equivalently,

$$
\Phi_{x}\left(\sigma_{x}(g)\right)=E_{x} \Phi[g]
$$

This generalizes the older notion of quotient function, which was characterized by $\Phi_{x}\left(E_{x} g\right)=E_{x} \Phi[g]$. See [2]. This older version of the quotient function need not exist, even for simply connected domains $V$. Again see [2].

Consider the case of an analytic map $\Phi$ which is an equivalence of a simply connected domain $U$ onto a simply connected domain $V$. For each $x \in X$ we define a map $\Phi^{x}: S_{x}(U) \rightarrow S_{x}(V)$. Given $g \in U$ there is a ball $b(g)=B(g, \epsilon)$ which is contained in $B_{U}(g)$ and whose image under $\Phi$ is contained in $B_{V}(\Phi[g])$. Put $\delta[x, g]=\sigma_{x}(b(g))$. The set $\delta[x, g]$ is a subdisk of $\Delta[x, g]$ in $S_{x}(U)$. Lemma 2.2 and the fact that $\sigma_{x}(g) \in \delta[x, g]$ show that the disks $\delta[x, g], g \in V$, cover $S_{x}(U)$. As before let $\Phi[f]=\Sigma g_{n}(f-g)^{n}$ be the power series expansion of $\Phi$ at g. Define $\Phi^{x}$ on $\delta[x, g]$ by the conditions

$$
P \Phi^{x}\left(\sigma_{x}(g)+z\right)=\sum_{n=0}^{\infty} g_{n}(x) z^{n}, \text { that is, } P \Phi^{x}=\Phi_{x}
$$

and

$$
\Phi^{x}\left(\sigma_{x}(g)+z\right) \in \Delta[x, \Phi[g]] \subset S_{x}(V) .
$$

It is possible to satisfy these conditions because

$$
\begin{aligned}
P \Phi^{x}(\delta[x, g]) & =\Phi_{x}(\delta[x, g])=\Phi_{x}\left(\sigma_{x}(b(g))\right) \\
& =E_{x} \Phi[b(g)] \subset E_{x}{ }^{B}(\Phi[g])=P \Delta[x, \Phi[g]] .
\end{aligned}
$$

Lemma 3.2. The map $\Phi^{x}$ is an equivalence of $S_{x}(U)$ onto $S_{x}(V)$.

Proof. We show first that $\Phi^{x}$ is single valued, hence holomorphic. Let $g$ and $b$ be any two functions in $U$. Choose functions $g=f_{0}, f_{1}, \cdots, f_{n}=b$ in $U$ so 
that $f_{i} \in b\left(f_{i-1}\right)$. Proceed as in the proof of Lemma 3.1 to the application of the monodromy theorem, which implies that $\Phi^{x}$ is single valued.

Condition (3.4) with $z=0$ says $P \Phi^{x}\left(\sigma_{x}(g)\right)=\Phi[g](x)$. Since $P$ in one-to-ose on $\Delta[x, \Phi[g]]$ and $P \sigma_{x}(\Phi[g])=\Phi[g](x)$, we have

$$
\Phi^{x}\left(\sigma_{x}(g)\right)=\sigma_{x}(\Phi[g]) .
$$

Being an equivalence of $U$ onto $V, \Phi$ has an analytic inverse $\Psi: V \rightarrow U$. The map $\Psi$ induces $\Psi^{x}: S_{x}(V) \rightarrow S_{x}(U)$ with the property $\Psi^{x} \sigma_{x}=\sigma_{x} \Psi$. By Lemma 2.2 every point of $S_{x}(U)$ has the form $\sigma_{x}(g)$ for some $g \in U$, and

$$
\Psi^{x} \Phi^{x}\left(\sigma_{x}(g)\right)=\Psi^{x} \sigma_{x}(\Phi[g])=\sigma_{x}(\Psi \Phi[g])=\sigma_{x}(g) .
$$

Likewise $\Phi^{x} \Psi^{x}$ is the identity on $S_{x}(V)$. It follows that $\Phi^{x}$ is an equivalence of $S_{x}(U)$ onto $S_{x}(V)$.

We call $\Phi^{x}$ the section mapping of $\Phi$ at the point $x$. One result of Lemma 3.2 is that if $U$ and $V$ are equivalent, then their corresponding sections must be equivalent. Other conditions for equivalence will come from looking at all the sections simultaneously.

Write $S(U)$ for the disjoint union of all the sections $S_{x}(U), x \in X$. Each $f \in U$ induces a function $S f: X \rightarrow S(U)$ according to the rule $S f(x)=\sigma_{x}(f)$. The function $S f$ satisfies

(1) $S f(x) \in \Delta[x, f]$ for all $x$, and

(2) $P S f=f$ is continuous.

Put $W(U)=\{S f ; f \in U\}$. Write $W^{*}(U)$ for the collection of functions $f: X \rightarrow S(U)$ which satisfy

(1') each point $y \in X$ has a neighborhood $Y$ and an associated function $b \in U$ so that $x \in Y$ implies $f(x) \in \Delta[x, b]$, and

$\left(2^{\prime}\right)$ the function $P f: X \rightarrow C$ is continuous.

We say $U$ is full if $W^{*}(U)=W(U)$.

Lemma 3.3. If $U$ and $V$ are equivalent simply connected domains and $U$ is full, then $V$ is full.

Proof. Let $\Phi$ be an equivalence of $U$ onto $V$ and let $\Psi$ be the inverse equivalence of $V$ onto $U$. Fix a function $f \in W^{*}(V)$ and put $g(x)=\Psi^{x}(f(x))$, $x \in X$. This makes sense because by $\left(1^{\prime}\right)$ we have $f(x) \in S_{x}(V)$. The function value $g(x)$ belongs to $S_{x}(U)$. We show that $g$ belongs to $W^{*}(U)$.

Given a point $y \in X$, choose a neighborhood $Y$ of $y$ and a function $b \in V$ so that $x \in Y$ implies $f(x) \in \Delta[x, b]$. In particular $f(y) \in \Delta[y, b]$; therefore $f(y)=\sigma_{y}(b+z)$ for some complex number $z$. Put $b^{\prime}=b+z$; notice that $b^{\prime} \in B_{V}(b)$. Choose a ball $b\left(b^{\prime}\right)$ about $b^{\prime}$ so that $b\left(b^{\prime}\right) \subset B_{V}(b)$ and $\Psi$ maps 
$b\left(b^{\prime}\right)$ into $B_{U}\left(\Psi\left[b^{\prime}\right]\right)$. Put $\delta\left[x, b^{\prime}\right]=\sigma_{x}\left(b\left(b^{\prime}\right)\right)$. Lemma 2.1 shows that $\delta\left[x, b^{\prime}\right] \subset$ $\Delta[x, b]$ for all $x$. By $\left(2^{\prime}\right)$ the point $y$ has a neighborhood $Y^{\prime}$ on which $P f(x)$ differs from $b^{\prime}(y)=P f(y)$ by less than half the radius of $b\left(b^{\prime}\right)$. Since $b^{\prime}$ is continuous, $y$ also has a neighborhood $Y^{\prime \prime}$ on which $b^{\prime}(x)$ differs from $b^{\prime}(y)$ by less than half the radius of $b^{\prime}\left(b^{\prime}\right)$. For $x \in Y \cap Y^{\prime} \cap Y^{\prime \prime}$ it follows that $f(x) \epsilon$ $\delta\left[x, b^{\prime}\right]$. Condition (3.5) in the definition of $\Psi^{x}$ shows that $g(x)=\Psi^{x}(f(x))$ belongs to $\Delta\left[x, \Psi\left[b^{\prime}\right]\right]$ when $x \in Y \cap Y^{\prime} \cap Y^{\prime \prime}$.

To show that $P g$ is continuous at $y$ let $\Psi^{*}$ be the restriction of $\Psi$ to $b\left(b^{\prime}\right)$. The section of $b\left(b^{\prime}\right)$ at $x$ is the disk centered at $b^{\prime}(x)$ with radius equal to the radius of $b\left(b^{\prime}\right)$. The mapping $\Psi_{x}^{*}$ is the quotient function of $\Psi^{*}$ at $x$ in the older sense, as well as the newer. Lemma 3 in [10] shows that as $x \rightarrow y$ the function $\Psi_{x}^{*}$ converges uniformly to $\Psi_{y}^{*}$ inside a neighborhood of $b^{\prime}(y)$. For $x \in Y \cap Y^{\prime} \cap Y^{\prime \prime}$ we have

$$
P_{g}(x)=P \Psi^{x}(f(x))=\Psi_{x}(f(x))=\Psi_{x}^{*}(P f(x)) .
$$

By $\left(2^{\prime}\right)$ the function $P f$ is continuous. Since $P f(y)=b^{\prime}(y)$, we see that $P g$ is continuous at $y$.

We have shown that $g$ satisfies $\left(1^{\prime}\right)$ and $\left(2^{\prime}\right)$. Since $U$ is full, there is a function $g^{\prime} \in U$ with $\sigma_{x}\left(g^{\prime}\right)=g(x)$ for all $x$. Hence

$$
\sigma_{x}\left(\Phi\left[g^{\prime}\right]\right)=\Phi^{x}\left(\sigma_{x}\left(g^{\prime}\right)\right)=\Phi^{x}(g(x))=\Phi^{x} \Psi^{x}(f(x))=f(x) .
$$

In other words $f=S \Phi\left[g^{\prime}\right]$ belongs to $W(V)$. This shows that $W^{*}(V) \subset W(V)$. The inclusion $W(V) \subset W^{*}(V)$ is always true. Thus $W(V)=W^{*}(V)$, and the lemma is proved.

Any ball $B(f, \epsilon)$ is full. This is because the section of $B(f, \epsilon)$ at $x$ is $D(f(x), \epsilon)$. If $g$ is continuous and $g(x) \in D(f(x), \epsilon)$ for all $x$, then $\|g-f\|<\epsilon$, that is, $g \in B(f, \epsilon)$. Suppose $V=\bigcup\{B(z, 1 / 2) ; z \in C,|z|<1 / 2\}$. If the space $X$ consists of more than one point, then the domain $V$ is not full, even though its sections are the same as those of the unit ball $B$.

4. Sections and continuity. We are interested in comparing $S_{x}(V)$ with $s_{y}(V)$ as $x \rightarrow y$. To begin with we give rules for how a subset of one section should translate into another section. We assume $V$ is a simply connected domain that contains the zero function.

Let $\mathcal{O}$ be a domain in $S_{x}(V)$ that contains $\sigma_{x}(0)$. We say $T: \mathcal{O} \rightarrow S_{y}(V)$ is a translation of $\Theta$ into $S_{y}(V)$ if

(1) $P T w=P w$ for all $w \in O$;

(2) $T \sigma_{x}(0)=\sigma_{y}(0)$;

(3) $T$ is continuous;

(4) $T$ is one-to-one. 
Such a translation need not exist, but if it does we say $\mathcal{O}$ translates into $s_{y}(V)$.

Lemma 4.1. If $\Theta$ translates into $S_{y}(V)$, then the translation $T$ is unique and bolomorphic.

Proof. Let $w$ be any point of $\mathcal{C}$. Choose disks $\Delta[x, f]$ and $\Delta[y, g]$ with $w \in \Delta[x, f]$ and $T w \in \Delta[y, g]$. By (3) there is a neighborhood $N$ of $w$ in $\Delta[x, f]$ such that $T(N) \subset \Delta[y, g]$. The projection $P$ is a local uniformizing parameter on both $\Delta[x, f]$ and $\Delta[y, g]$; therefore (1) shows that $T$ is holomorphic at $w$, hence holomorphic. Putting $w=\sigma_{x}(0)$, we see from (2) that $T$ is completely determined on a neighborhood $N$ of $\sigma_{x}(0)$. Since $\mathcal{O}$ is connected, the uniqueness theorem for holomorphic functions shows $T$ is unique.

We say that a set $E \subset S_{x}(V)$ translates into $S_{y}(V)$ if $E$ is contained in a domain $\mathcal{O}$ which translates into $S_{y}(V)$.

We examine the possibility of translation when $V$ is equivalent to the unit ball $B$. For every $x$ the section of $B$ at $x$ is the unit disk $D=D(0,1)$. For $r>0$ put $K(r)=\{z \in C ;|z| \leq r\}$.

Lemma 4.2. Let $\Phi$ be an equivalence of $B$ onto $V$ with $\Phi[0]=0$. For each $y \in X$ and $r_{0} \in(0,1)$, there is a neighborbood $Y$ of $y$ so that $x \in Y$ implies $\Phi^{y}\left(K\left(r_{0}\right)\right)$ translates into $S_{x}(V)$ and $\Phi^{x}\left(K\left(r_{0}\right)\right)$ translates into $S_{y}(V)$.

Proof. Lemma 3.2 shows that $\Phi^{x}$ is an equivalence of $D$ onto $S_{x}(V)$. Thus the quotient function $\Phi_{x}$ is locally one-to-one on $D$. Choose $r_{1}$ and $r_{2}$ with $r_{0}<r_{1}<r_{2}<1$. Put $\delta_{1}=\inf \left\{|z-w| ; z \neq w, \Phi_{y}(z)=\Phi_{y}(w)\right.$ and $\left.|z|,|w| \leq r_{2}\right\}$. We have $\delta_{1}>0$ because $\Phi_{y}$ is locally one-to-one. Put $\epsilon=1 / 2 \min \left\{\delta_{1}, r_{2}-r_{1}\right\}$. The quantity $\delta_{2}=\inf \left\{\left|\Phi_{y}(z)-\Phi_{y}(w)\right| ;|z-w|=\epsilon\right.$ and $\left.|z|,|w| \leq r_{2}\right\}$ is positive. Lemma 3 in [10] states that $\Phi_{x}$ converges uniformly inside $D$ to $\Phi_{y}$ as $x \rightarrow y$. In particular $y$ has a neighborhood $Y$ so that $\left|\Phi_{x}(z)-\Phi_{y}(z)\right|<1 / 4 \delta_{2}$ whenever $x \in Y$ and $z \in K\left(r_{2}\right)$. It follows from Rouche's theorem that if $x \in Y$ and $z \in K\left(r_{1}+\epsilon\right)$ there are unique complex numbers $a(x, z)$ and $b(x, z)$ satisfying

$$
\begin{aligned}
& |z-a(x, z)|<\epsilon \text { and } \Phi_{x}(a(x, z))=\Phi_{y}(z), \\
& |z-b(x, z)|<\epsilon \text { and } \Phi_{y}(b(x, z))=\Phi_{x}(z) .
\end{aligned}
$$

In checking (4.2) observe that $2 \epsilon \leq \delta_{1}$ and that if $|z-w|=\epsilon$ then $\left|\Phi_{x}(z)-\Phi_{x}(w)\right|$ $\geq 1 / 2 \delta_{2}$ for $x \in Y$ and $z, w \in K\left(r_{2}\right)$.

Put $\mathcal{O}_{y}=\Phi^{y}\left(D\left(0, r_{1}\right)\right)$. For $x \in Y$ define $T: \Theta_{y} \rightarrow S_{x}(V)$ by

$$
T \Phi^{y}(z)=\Phi^{x}(a(x, z)) \text {. }
$$

We show that $T$ satisfies all the conditions for a translation. Condition (1) follows 
from the second parts of (3.4) and (4.1). From the hypothesis $\Phi[0]=0$ we get $\Phi_{x}(0)=0$ and hence $a(x, 0)=0$ for all $x$. Condition (2) holds because

$$
T \sigma_{y}(0)=T \Phi^{y}(0)=\Phi^{x}(a(x, 0))=\Phi^{x}(0)=\sigma_{x}(0) .
$$

To verify (3) fix a point $z_{0} \in D\left(0, r_{1}\right)$ and consider the branch of $\Phi_{x}^{-1}$ which satisfies $a\left(x_{0} z_{0}\right)=\Phi_{x}^{-1} \Phi_{y}\left(z_{0}\right)$. This inverse function is continuous in a neighborhood of $\Phi_{y}\left(z_{0}\right)$; therefore $a(x, z)$ is continuous at $z=z_{0}$. Since $\Phi^{y}$ and $\Phi^{x}$ are equivalences of $D$ onto $S_{y}(V)$ and $S_{x}(V)$ respectively, $T$ is continuous.

Suppose $T \Phi^{y}(z)=T \Phi^{y}(w)$. From the definition of $T$ we get $\Phi^{x}(a(x, z))=$ $\Phi^{x}(a(x, w))$, and since $\Phi^{x}$ is one-to-one, $a(x, z)=a(x, w)$. Line (4.1) shows that $|z-w|<2 \epsilon$ and $\Phi_{y}(z)=\Phi_{y}(w)$. Since $2 \epsilon \leq \delta_{1}$, the definition of $\delta_{1}$ implies $z=w$. Thus (4) holds, and $T$ is a translation.

For $x \in Y$ put $\mathcal{O}_{x}=\Phi^{x}\left(D\left(0, r_{1}\right)\right)$. Define $T: \mathcal{O}_{x} \rightarrow S_{y}(V)$ by $T \Phi^{x}(z)=$ $\Phi^{y}(b(x, z))$. The argument that $T$ is a translation is the same as above until we come to condition (4). Suppose $z, w \in D\left(0, r_{1}\right)$ and $T \Phi^{x}(z)=T \Phi^{x}(w)$. The definition of $T$ and the one-to-one property of $\Phi^{y}$ imply $b(x, z)=b(x, w)=\zeta$. From (4.2) we get $|\zeta-z|<\epsilon,|\zeta-w|<\epsilon$ and $\Phi_{x}(z)=\Phi_{y}(\zeta), \Phi_{x}(w)=\Phi_{y}(\zeta)$. The function $a(x, \zeta)$ is defined because $\zeta \in K\left(r_{1}+\epsilon\right)$. By $(4.1), z=a(x, \zeta)=w$.

We have $\Phi^{y}\left(K\left(r_{0}\right)\right) \subset \theta_{y}$ and for $x \in Y, \Phi^{x}\left(K\left(r_{0}\right)\right) \subset \Theta_{x}$. This proves the lemma.

Let $V$ be a simply connected domain in $C(X)$ with $0 \in V$. Each section $S_{x}(V)$ is an open simply connected Riemann surface. The surface is open because $P\left(s_{x}(V)\right)=V_{x}$ is an open subset of the finite plane. It is known [1] that such a Riemann surface is equivalent either to the plane or to the disk. Assume that $S_{x}(V)$ is always equivalent to the disk. In this case there is a unique equivalence $F^{x}$ of $D$ onto $S_{x}(V)$ which satisfies $F^{x}(0)=\sigma_{x}(0)$ and $\left(P F^{x}\right)^{\prime}(0)>0$. Put $F_{x}=P F^{x}$. The Riemann surface of the holomorphic function $F_{x}$ is $S_{x}(V)$.

Definition. We say $x \rightarrow S_{x}(V)$ is continuous at $x=y$ if for each $r \in(0,1)$ there is a neighborhood $Y$ of $y$ for which $x \in Y$ implies $F^{y}(K(r))$ translates into $S_{x}(V)$ and $F^{x}(K(r))$ translates into $S_{y}(V)$. In this case we also say $S_{x}(V)$ converges to $S_{y}(V)$ as $x \rightarrow y$.

If all the sections $S_{y}(V)$ are single sheeted, then this definition is equivalent to the Carathédory notion of convergence of domains. For two versions of Carathéodory's definition see [3] and [4]. Roughly speaking, the possibility of translating $F^{y}(K(r))$ into $S_{x}(V)$ means that $S_{y}(V)$ is not too big. The possibility of translating $F^{x}(K(r))$ into $S_{y}(V)$ means that $S_{y}(V)$ is not too small.

Lemma 4.3. If $S_{x}(V)$ converges to $S_{y}(V)$ as $x \rightarrow y$, then $F_{x}$ converges uniformly inside $D$ to $F_{y^{*}}$

Proof. For $n=2,3$, etc., put $D_{n}=D\left(0,1-n^{-1}\right)$ and let $K_{n}$ be the closure 
of $D_{n}$. Let $A$ be any directed set so that the net $\{x(a) ; a \in A\}$ converges in $X$ to $\cdot y$. With respect to nets we use the terminology of $\mathrm{Hu}$ [6]. For each $n$ the set $F^{y}\left(K_{n}\right)$ eventually translates into $S_{x(\alpha)}(V)$ and $F^{x(a)}\left(K_{n}\right)$ eventually translates into $S_{y}(V)$.

Let $G_{x}$ be the inverse of $F^{x}$, that is, $G_{x}$ is the equivalence of $S_{x}(V)$ onto $D$ with $G_{x}\left(\sigma_{x}(0)\right)=0$ and $G_{x}^{\prime}\left(\sigma_{x}(0)\right)>0$. The function $H_{a}=G_{y} T F^{x(a)^{x}}$ eventually maps $D_{n}$ holomorphically into $D$ with $H_{a}(0)=0$ and $H_{a}^{\prime}(0)>0$. Being uniformly bounded, the family $\left\{H_{a} ; a \in A\right\}$ is eventually normal in each $D_{n}$, hence eventually normal on $D$. Let $H$ be an accumulation point of $\left\{H_{a} ; a \in A\right\}$ in the topology of uniform convergence on compact subsets of $D$. There is a subnet $A^{*}$ of $A$ with $\left\{H_{\beta} ; \beta \in A^{*}\right\}$ converging to $H$. In particular $H: D \rightarrow D$ and $H(0)$ $=\lim H_{\beta}(0)=0, H^{\prime}(0)=\lim H_{\beta}^{\prime}(0) \geq 0$.

For each $n$ eventually $H_{\beta}$ maps $D_{n}$ into $D$, with $H_{\beta}(0)=0$. The Schwarz lemma, shows that eventually $H_{\beta}^{\prime}(0) \leq n /(n-1)$. On the other hand, the function $J_{\beta}=G_{x(\beta)} T F^{y}$ eventually maps $D_{n}$ into $D$ with $J_{\beta}(0)=0$. By the Schwarz lemma $J_{\beta}^{\prime}(0) \leq n /(n-1)$. We have

$$
J_{\beta}^{\prime}(0)=G_{x(\beta)}^{\prime}\left(\sigma_{x(\beta)}(0)\right) F_{y}^{\prime}(0)=\left\{F_{x(\beta)}^{\prime}(0) G_{y}^{\prime}\left(\sigma_{y}(0)\right)\right\}^{-1}=\left\{H_{\beta}^{\prime}(0)\right\}^{-1}
$$

thus for each $n$ eventually

$$
(n-1) / n \leq H_{\beta}^{\prime}(0) \leq n /(n-1) .
$$

This makes $H^{\prime}(0)=1$. $\Lambda$ corollary to the Schwarz lemma [5] implies that $H(z)=z$. In other words the subnet $\left\{H_{\beta} ; \beta \in A^{*}\right\}$ converges uniformly inside $D$ to the identity.

Since $F_{y}$ is continuous on $D$, the subnet $\left\{F_{x(\beta)}=F_{y} H_{\beta} ; \beta \in A^{*}\right\}$ converges uniformly inside $D$ to $F_{y^{\circ}}$.

We finish the proof of this lemma by a contradiction argument. Suppose $F_{x}$ did not converge uniformly inside $D$ to $F_{y}$ as $x \rightarrow y$. In that case there would be a compact $K$, and $\epsilon>0$, a net $\{x(\alpha) ; a \in A\}$ converging to $y$ and points $z_{a} \in K$ satisfying $\left|F_{x(\alpha)}\left(z_{a}\right)-F_{y}\left(z_{\alpha}\right)\right| \geq \epsilon$ for all $a$. Construct the subnet $\left\{F_{x(\beta)} ; \beta \in A^{*}\right\}$ as above. This subnet converges to $F_{y}$ uniformly on $K$. Eventually $\left|F_{x(\beta)}\left(z_{\beta}\right)-F_{y}\left(z_{\beta}\right)\right|<\epsilon$, which is a contradiction.

The following result is needed for Theorem 5.3. It also shows how disks $\Delta[x, b], b \in V$, behave under translation.

Lemma 4.4. If $b \in V$ and if $x \rightarrow S_{x}(V)$ is continuous at $x=y$, then for $x$ in some neighborbood of $y$ the point $\sigma_{y}(b) \in S_{y}(V)$ translates into $\Delta[x, b] \subset S_{x}(V)$.

Proof. Choose functions $0=b_{0}, b_{1}, \ldots, b_{n}=b$ in $V$ with $B\left(b_{i-1}\right) \cap B\left(b_{i}\right)$ 
nonempty for $i=1, \ldots, n$. Let $\eta$ be the path $\left[b_{0}, b_{1}\right]+\cdots+\left[b_{n-1}, b_{n}\right]$. The path $\sigma_{y} \eta$ is compact in $S_{y}(V)$ and is contained in $F^{y}(K(r))$ for some $r \epsilon(0,1)$. The continuity of $x \rightarrow S_{x}(V)$ at $x=y$ means $F^{y}(K(r))$ translates into $S_{x}(V)$ for $x$ in some neighborhood $Y_{1}$ of $y$. For $x \in Y_{1}$ the path $\sigma_{y} \eta$ translates into $S_{x}(V)$.

Recall that $D\left[x, b_{i}\right]=E_{x}\left(B\left(b_{i}\right)\right)$. Use the continuity of $b_{0}, b_{1}, \ldots, b_{n}$ to choose a neighborhood $Y_{2}$ of $y$ on which $b_{i}(y) \in D\left[x, b_{i}\right]$ and $\left[b_{i-1}(y), b_{i}(y)\right] \subset$ $D\left[x, b_{i-1}\right] \cup D\left[x, b_{i}\right]$. Put $Y=Y_{1} \cap Y_{2}$.

For $x \in Y$ let $T$ be the translation of $\sigma_{y} \eta$ into $S_{x}(V)$. The translated path is polygonal, and its vertices are the points $T \sigma_{y}\left(b_{i}\right)$. We show by induction that $T \sigma_{y}\left(b_{i}\right) \in \Delta\left[x_{,} b_{i}\right]$. This is true for $i=0$ because $b_{0}=0$ and $T \sigma_{y}(0)=\sigma_{x}(0)$. Suppose $T \sigma_{y}\left(b_{i-1}\right) \in \Delta\left[x, b_{i-1}\right]$. Since $B\left(b_{i-1}\right) \cap B\left(b_{i}\right) \neq \varnothing$, the projection $P$ is an equivalence of $\Delta\left[x, b_{i-1}\right] \cup \Delta\left[x, b_{i}\right]$ onto $D\left[x, b_{i-1}\right] \cup D\left[x, b_{i}\right]$. See Lemma 2.1. Since $P T=P, P$ sends $T\left[\sigma_{y}\left(h_{i-1}\right), \sigma_{y}\left(h_{i}\right)\right]$ one-to-one onto $\left[b_{i-1}(y), b_{i}(y)\right]$. By choice of $Y_{2}$ this segment lies in $D\left[x, b_{i-1}\right] \cup D\left[x, b_{i}\right]$, so it is the image under $P$ of a segment in $\Delta\left[x, b_{i-1}\right] \cup \Delta\left[x, b_{i}\right]$. One end of the latter segment is $T \sigma_{y}\left(b_{i-1}\right)$; the other end is $\operatorname{To}_{y}\left(b_{i}\right)$. Since $b_{i}(y) \in D\left[x, b_{i}\right]$, we have $T \sigma_{y}\left(b_{i}\right) \in \Delta\left[x, b_{i}\right]$. By induction $T \sigma_{y}(b) \in \Delta[x, b]$.

5. Domains equivalent to $B$. We know the following conditions are necessary for a domain $V$ to be equivalent to $B$ :

(5.1) $V$ is simply connected;

(5.2) each section of $V$ is equivalent to the disk;

(5.3) $x \rightarrow S_{x}(V)$ is continuous at each point of $X$;

(5.4) $V$ is full.

The first of these is necessary because every equivalence is a homeomorphism. The others follow from Lemma 3.2, Lemma 4.2, and Lemma 3.3 respectively.

We now assume that $V$ is a domain in $C(X)$ which satisfies (5.1) through (5.4). We also assume $V$ contains the zero function. Our aim is to prove that $V$ is equivalent to $B$. As standard notation we let $F^{x}$ be the unique equivalence of $D$ onto $S_{x}(V)$ with $F^{x}(0)=\sigma_{x}(0)$ and $F_{x}^{\prime}(0)>0$, where $F_{x}=P F^{x}$.

Lemma 5.1. The function $\Phi$ defined by $\Phi[f](x)=F_{x}(f(x))$ maps $B$ analytically into $C(X)$.

Proof. Each function $F_{x}$ is an ordinary holomorphic function on $D$. It has a power series expansion

$$
F_{x}(z)=\sum_{n=1}^{\infty} a_{n}(x) z^{n}
$$

that converges for $|z|<1$. The coefficient $a_{n}(x)$ is given by 


$$
a_{n}(x)=r^{-n} \int_{0}^{2 \pi} F_{x}\left(r e^{i \theta}\right) e^{-i n \theta} d(\theta / 2 \pi)
$$

for each $r$ satisfying $0<r<1$. Condition (5.3) and Lemma 4.3 show that for each $r$ in this interval $F_{x}\left(r e^{i \theta}\right)$ converges uniformly in $\theta$ to $F_{y}\left(r e^{i \theta}\right)$ as $x \rightarrow y$. It follows that $a_{n}(x)$ is a continuous function of $x$, that is, $a_{n} \in C(X)$. Likewise $\sup _{\theta}\left|F_{x}\left(r e^{i \theta}\right)\right|$ is a continuous function of $x$, and it has a maximum value $M(r)$ on the compact space $X$. Equation (5.6) gives us $\left\|a_{n}\right\| \leq r^{-n} M(r)$. This is true for $0<r<1$; therefore $\left(\lim \sup \left\|a_{n}\right\|^{1 / n}\right)^{-1} \geq 1$. This inequality implies that the power series $\Sigma a_{n} f^{n}$ converges when $\|f\|<1$, that is, when $f \in B$. In particilar $\Sigma a_{n} f^{n}$ is a continuous function. By the definition of $\Phi$ and (5.5)

$$
\Phi[f](x)=F_{x}(f(x))=\sum_{n=1}^{\infty} a_{n}(x)(f(x))^{n} .
$$

Thus $\Phi[f]=\Sigma a_{n} f^{n}$, and $\Phi$ is analytic on $B$.

Lemma 5.2. If $f \in B$ and $g(x)=F^{x}(f(x))$, then $g \in W^{*}(V)$.

Proof. The function $P g: X \rightarrow C$ is continuous because $P g(x)=P F^{x}(f(x))=$ $F_{x}(f(x))=\Phi[f](x)$ and $\Phi[f]$ is continuous. For all $x$ the function value $g(x)$ belongs to $S_{x}(V)=F^{x}(D)$. Fix a point $y \in X$. By Lemma 2.2 there is an $b \in V$ with $\sigma_{y}(b)=g(y)$. We show that $g(x) \in \Delta[x, b]$ for $x$ in some neighborhood of $y$, and so conclude that $g \in W^{*}(V)$.

Modify the proof of Lemma 4.2. As a first step we everywhere substitute $F_{x}$ for $\Phi_{x}$ and $F^{x}$ for $\Phi^{x}$. This is permissible because $F^{x}$ is an equivalence of $D^{x}$ onto $S_{x}(V), F^{x}(0)=\sigma_{x}(0)$ and $F_{x}$ converges uniformly inside $D$ to $F_{y}$ as $x \rightarrow y$. Choose $r_{0}=|f(y)|$, and proceed to choose the neighborhood $Y$ and the functions $a(x, z), b(x, z)$. For $x \in Y$ and $z \in K\left(r_{1}+\epsilon\right)$ we have

$$
a(x, z)=F_{x}^{-1} F_{y}(z) \text { and } b(x, z)=F_{y}^{-1} F_{x}(z)
$$

where $F_{x}^{-1}$ and $F_{y}^{-1}$ are the appropriate branches of the inverse functions of $F_{x}$ and $F_{y}$. In particular the branches are chosen so that $a(x, b(x, z))=z$. Equations (5.7) and the uniform convergence of $F_{x}$ to $F_{y}$ show that $a$ and $b$ are continuous at $(x, z)=(y, f(y))$.

Let $T$ be the translation of $\mathcal{O}_{y}=F^{y}\left(D\left(0, r_{1}\right)\right)$ into $S_{x}(V)$. By (4.3) $T F^{y}(b(x, z))=F^{x}(a(x, b(x, z)))=F^{x}(z)$. In particular

$$
T F^{y}(b(x, f(x)))=F^{x}(f(x))=g(x) .
$$

By Lemma 4.4 we may shrink the neighborhood $Y$ so that, for $x \in Y, T F^{y}(f(y))=$ $T_{g}(y)=T \sigma_{y}(b) \in \Delta[x, b]$. Since $P T=P$ and since $b$ is continuous, we may further shrink $Y$ so that $T$ translates a fixed neighborhood $N$ of $\sigma_{y}(b)$ into 
$\Delta[x, b]$ when $x \in Y$. Shrink $Y$ for a third time to insure that $x \in Y$ implies $F^{y}(b(x, f(x))) \in N$. This is possible because $f$ and $F^{y}$ are continuous and $b$ is continuous at $(y, f(y))$. Equation (5.8) and $x \in Y$ imply $g(x) \in T(N) \subset \Delta[x, b]$.

Theorem 5.3. For $V$ and $B$ to be equivalent it is necessary and sufficient that $V$ satisfy (5.1) tbrough (5.4).

Proof. We have already remarked on the necessity of the conditions. If the conditions are satisfied, we claim that the analytic mapping $\Phi$ of Lemma 5.1 is an equivalence of $B$ onto $V$.

To show that $\Phi$ maps $B$ into $V$ let $f$ be any function in $B$ and let $g \in W^{*}(V)$ be the function of Lemma 5.2. By condition (5.4) there is an $b \in V$ with $\sigma_{x}(b)=g(x)$ for all $x$. Thus $b(x)=P \sigma_{x}(b)=P g(x)=\Phi[f](x)$. In other words $\Phi[f]=b$ belongs to $V$. As a byproduct we get the equation

$$
\sigma_{x}(\Phi[f])=\sigma_{x}(b)=F^{x}(f(x)) \text {. }
$$

This can be used to show that $\Phi$ is one-to-one. For if $\Phi[f]=\Phi[g]$, then by (5.9) $F^{x}(f(x))=\sigma_{x}(\Phi[f])=\sigma_{x}(\Phi[g])=F^{x}(g(x))$. For each $x$ the mapping $F^{x}$ is one-to-one; therefore $f(x)=g(x)$ for all $x$, that is, $f=g$.

We next show that $\Phi$ has an analytic inverse on $\Phi[B]$. Let $f$ be any function in $B$. The derivative of $\Phi$ at $f$ is the function $\Phi^{\prime}[f]$. The value of $\Phi^{\prime}[f]$ at $x \in X$ is

$$
\Phi^{\prime}[f](x)=\sum_{n=1}^{\infty} n a_{n}(x)(f(x))^{n-1}=F_{x}^{\prime}(f(x)) \neq 0 .
$$

The derivative on the right is not zero because $F_{x}$ is locally one-to-one. Thus $\Phi^{\prime}[f]$ is nowhere vanishing and has a multiplicative inverse in $C(X)$. It follows [2] that $\Phi[B]$ is a domain and that the inverse of $\Phi$ is analytic. Call the inverse mapping $\Psi$.

We have shown that $\Phi$ is an equivalence of $B$ onto $\Phi[B]$ and that $\Phi[B] \subset V$. By Lemma 3.2 the section map $\Phi^{x}$ is an equivalence of $D=S_{x}(B)$ into $S_{x}(\Phi[B])$. Since $P \Phi^{x}=\Phi_{x}$, the surface $S_{x}(\Phi[B])$ is the Riemann surface of the holomorphic function $\Phi_{x}$ on $D$. In the same way $S_{x}(V)=F^{x}(D)$ is the Riemann surface of $F_{x}$ on $D$. Together (3.1), (5.5) and the expansion $\Phi[f]=\Sigma a_{n} f^{n}$ imply that $\Phi_{x}=F_{x}$; therefore $S_{x}(\Phi[B])=S_{x}(V)$.

Let $f$ be any function in $V$ and put $S f(x)=\sigma_{x}(f)$. We show that $S f$ belongs to $W^{*}(\Phi[B])$. For each $x$ we have $S f(x) \in S_{x}(\Phi[B])$ because $\sigma_{x}(f) \in S_{x}(V)=$ $S_{x}(\Phi[B])$. The function $P S f=f$ is continuous. Let $y$ be a point in $X$. By Lemma 2.2 there is an $b \in \Phi[B]$ with $\sigma_{y}(b)=S f(y)$. By condition (5.3) the point $\sigma_{y}(b)=$ $S f(y)$ translates, into $S_{x}(\Phi[B])=S_{x}(V)$ for $x$ in some neighborhood $Y$ of $y$. For 
a given $x \in Y$ let $T$ be this translation. By Lemma 4.4 we can shrink $Y$ so that $x \in Y$ implies

$$
T \sigma_{y}(b)=T S f(y)=T \sigma_{y}(f) \in \Delta[x, b] \cap \Delta[x, f] .
$$

Using $P T=P$ and the continuity of $b$ and $f$, shrink $Y$ further and choose an open disk $N$ about $\sigma_{y}(b)=\sigma_{y}(f)$ to achieve $T(N) \subset \Delta[x, b] \cap \Delta[x, f]$ and $f(x) \epsilon$ $P(N)$ for $x \in Y$. For all $x$ the projection $P$ is one-to-one on $\Delta[x, f]$, the point $S f(x)$ belongs to $\Delta[x, f]$ and $P S f(x)=f(x)$. Thus, for $x \in Y, S f(x) \in T(N) \subset$ $\Delta[x, b]$. It follows that $S f \in W^{*}(\Phi[B])$.

By Lemma 3.3 the domain $\Phi[B]$ is full; therefore $S f=S_{g}$ for some $g \in \Phi[B]$. This gives us $f=P S f=P S g=g \in \Phi[B]$. Hence $V=\Phi[B]$, and $V$ is equivalent to $B$.

6. Continuously tubular domains. We consider here the effect of removing condition (5.2), that is, of allowing $S_{x}(V)$ to be equivalent to the plane. As an example let $r: X \rightarrow(0, \infty]$ be continuous and put $U(r)=\{f \in C(X) ;|f(x)|<r(x)\}$. The domains $U(r)$ are simple representatives of equivalence classes of domains, just as $B=U(1)$ is such a representative. We shall show that $(5.1),(5.3)$ and (5.4) characterize the domains equivalent to some $U(r)$ if the definition of (5.3) is modified.

Let $V$ be a simply connected domain in $C(X)$, and assume that $V$ contains the zero function. Each section $S_{x}(V)$ has an interior mapping radius $r(x)$ with respect to $\sigma_{x}(0)$. The section $S_{x}(V)$ is equivalent to the plane precisely when $r(x)=\infty$. Let $F^{x}$ be the unique equivalence of $D(0, r(x))$ onto $S_{x}(V)$ with $F^{x}(0)=\sigma_{x}(0)$ and $F_{x}^{\prime}(0)=1$, where $F_{x}=P F^{x}$. We shall also use the mapping $G_{x}: S_{x}(V) \rightarrow D(0, r(x))$ which is inverse to $F^{x}$.

Definition. We say $x \rightarrow S_{x}(V)$ is continuous at $x=y$ if the radius function $r$ is continuous at $y$ and if for each $r_{0} \in(0, r(y))$ there is a neighborhood $Y$ of $y$ with the property that when $x \in Y$

(1) $F^{y}\left(K\left(r_{0}\right)\right)$ translates into $S_{x}(V)$,

(2) $F^{x}\left(K\left(r_{0}\right)\right)$ translates into $S_{y}(V)$.

Note that by itself the continuity of $r$ implies $F^{x}$ is defined on $K\left(r_{0}\right)$ when $x$ is close to $y$.

Lemma 6.1. If $r$ is continuous and $F_{x}$ converges uniformly inside $D(0, r(y))$ to $F_{y}$ as $x \rightarrow y$, then $x \rightarrow S_{x}(V)$ is continuous at $x=y$.

Proof. Repeat the proof of Lemma 4.2 except write $r_{2}<r(y)$ in place of $r_{2}<1$ and write $F$ in place of $\Phi$.

Lemma 6.2. If $x \rightarrow S_{x}(V)$ is continuous at $x=y$, then $F_{x}$ converges uniformly inside $D(0, r(y))$ to $F_{y}$ as $x \rightarrow y$. 
Proof. As in the proof of Lemma 4.3 let $\{x(a) ; a \in A\}$ be a net converging to $y$. Our hypothesis implies that for any $r_{0}<r(y)$ the set $F^{x(\alpha)}\left(K\left(r_{0}\right)\right)$ eventually translates into $S_{y}(V)$. Thus $H_{\alpha}=G_{y} T F^{x(\alpha)}$ is eventually defined on $D\left(0, r_{0}\right)$. Observe that $F^{x(\alpha)}, T$ and $G_{y}$ are all holomorphic and oneto-one; the same is true of $H_{\alpha}$. Since $H_{\alpha}$ is a univalent map of $D\left(0, r_{0}\right)$ and since $H_{\alpha}(0)=0$ and $H_{a}^{\prime}(0)=1$, the Schwarz lemma shows that $H\left(D\left(0, r_{0}\right)\right)$ omits at least one complex number of modulus $r_{0}$. It follows from one of the Koebe distortion theorems [8] that $\left\{H_{\alpha} ; \alpha \in A\right\}$ is eventually normal on $D\left(0, r_{0}\right)$. Since $r_{0}<r(y)$ is arbitrary, $\left\{H_{a} ; a \in A\right\}$ is eventually normal on $D(0, r(y))$.

Being normal, the net $\left\{H_{\alpha} ; a \in A\right\}$ has an accumulation point $H$ and a subnet $\left\{H_{\beta} ; \beta \in A^{*}\right\}$ converging to $H$ in the topology of uniform convergence on compact subsets of $D(0, r(y))$. The functions $H_{\beta}$ are univalent with range in $D(0, r(y))$ and satisfy $H_{\beta}(0)=0, H_{\beta}^{\prime}(0)=1$; their limit $H$ has these properties too. Using the Schwarz lemma when $r(y)<\infty$ and Liouville's theorem when $r(y)=\infty$, one shows that $H(z)=z$. From here one follows the proof of Lemma 4.3.

Definition. A domain in $C(X)$ is called continuously tubular if it is equivalent to a domain of the form $U(r)$ where $r: X \rightarrow(0, \infty]$ is continuous. (This terminology is motivated by the case $X=[0,1]$.)

Theorem 6.3. Let $V$ be a domain containing the zero function. For $V$ to be continuously tubular it is necessary and sufficient that $V$ satisfy (5.1), (5.4), and the modified version of (5.3).

Proof of necessity. Assume that $r: X \rightarrow(0, \infty]$ is continuous and that $\Phi$ is an equivalence of $U(r)$ onto $V$. Condition (5.1) holds because $U(r)$ is simply connected and $\Phi$ is a homeomorphism. Lemma 3.3 and the fact that $U(r)$ is full imply (5.4). To show $x \rightarrow S_{x}(V)$ is continuous we first normalize $\Phi$ so that $\Phi[0]=0$. Suppose to begin with that $\Phi[g]=0, g \in U(r)$. Define $\Psi$ on $U(r)$ by

$$
\Psi[f]=(f-g) \sum_{n=0}^{\infty}\left(\bar{g} / r^{2}\right)^{n} f^{n} .
$$

Here $\bar{g}$ is the complex conjugate of $g$, and $\bar{g} / r^{2}$ vanishes when $r$ is infinite. The quotient function

$$
\Psi_{x}(z)=(z-g(x)) /\left(1-z \bar{g}(x) / r^{2}(x)\right)
$$

is a fractional linear transformation of $D(0, r(x))$ onto itself. The map $\Psi$ is an equivalence of $U(r)$ onto itself that sends $g$ to 0 . If we apply the inverse of $\Psi$ and then $\Phi$, we get an equivalence of $U(r)$ onto $V$ that sends 0 to 0 . Call this new equivalence $\Phi$, so that $\Phi[0]=0$.

By Lemma $3.2 \Phi^{x}$ is an equivalence of $D(0, r(x))$ onto $S_{x}(V)$. The normali- 
zation $\Phi[0]=0$ implies $\Phi^{x}(0)=\sigma_{x}(0)$. It follows that the interior mapping radius of $S_{x}(V)$ with respect to $\sigma_{x}(0)$ is $r(x)\left|\Phi^{\prime}[0](x)\right|$. This radius function is continuous because $r$ and $\Phi^{\prime}[0]$ are continuous. It is positive because $r$ is positive and $\Phi^{\prime}[0]$ is nonvanishing.

Put $s=r\left|\Phi^{\prime}[0]\right|$ and define $F$ on $U(s)$ by $F[f]=\Phi\left[f / \Phi^{\prime}[0]\right]$. The mapping $F$ is an equivalence of $U(s)$ onto $V$ satisfying $F[0]=0$ and $F^{\prime}[0]=1$. By Lemma $3.2, F^{x}$ is an equivalence of $D(0, s(x))$ onto $S_{x}(V)$, and $F^{x}(0)=\sigma_{x}(0)$ and $F_{x}^{\prime}(0)=1$. Fix $y \in X$. As a special case of Lemma 3 in [10] the quotient functions $F_{x}$ converge uniformly inside $D(0, s(y))$ to $F_{y}$ as $x \rightarrow y$. Lemma 6.1 shows that $x \rightarrow S_{x}$ is continuous at $x=y$. That is, the modified version of (5.3) holds.

Proof of sufficiency. Assume that $V$ satisfies (5.1), (5.4), and the modified version of (5.3). Let $r(x)$ be the interior mapping radius of $S_{x}(V)$ with respect to $\sigma_{x}(0)$. Let $F^{x}$ be the equivalence of $D(0, r(x))$ onto $S_{x}(V)$ with $F^{x}(0)=0$ and $F_{x}^{\prime}(0)=1$, where $P F^{x}=F_{x}$. The holomorphic function $F_{x}$ has a power series expansion $F_{x}(z)=\sum_{n=1}^{\infty} a_{n}(x) z^{n},|z|<r(x)$. The complex number $a_{n}(x)$ has the representation (5.6) whenever $0<r<r(x)$. Condition (5.3) and Lemma 6.2 show that $F_{x}$ converges uniformly on $\left\{z ;|z|=r_{0}\right\}$ to $F_{y}$ for each $r_{0}<r(y)$. It follows as in Lemma 5.1 that the functions $a_{n}$ belong to $C(X)$.

Define $\Phi$ on $U(r)$ by $\Phi[f](x)=\sum_{n=1}^{\infty} a_{n}(x)(f(x))^{n}$. The series converges for each $x$ because $f \in U(r)$ means $|f(x)|<r(x)$. We show $\Phi$ is analytic on $U(r)$ by showing it is analytic on a neighborhood of each function in $U(r)$. Given $f \in U(r)$, choose $\epsilon>0$ with $B(f, \epsilon) \subset U(r)$; thus $|f(x)|+\epsilon \leq r(x)$ for all $x$. Put $s(x)=$ $|f(x)|+\epsilon ; s$ is positive, continuous, and finite on $X$, and $f \in U(s) \subset U(x)$. Set $b_{n}=a_{n} s^{n}$. We have $b_{n} \in C(X)$, and for all $x$

$$
\left(\lim \sup \left|b_{n}(x)\right|^{1 / n}\right)^{-1} \geq r(x) / s(x) \geq 1 \text {. }
$$

Therefore $b \rightarrow \Sigma b_{n} b^{n}$ is analytic on $B$. On $U(s)$ the mapping $\Phi$ is the composition of $g \rightarrow g / s$ and $b \rightarrow \Sigma b_{n} b^{n}$. Hence $\Phi$ is analytic on $U(s)$.

The rest of the proof follows that of Theorem 5.3. In outline, condition (5.4) implies that $\Phi$ maps $U(r)$ into $V$. The fact that $\Phi$ is one-to-one comes from the one-to-one property of each section map $\Phi^{x}=F^{x}$. The inverse of $\Phi$ on $\Phi[U(r)]$ is analytic because $\Phi^{\prime}[f]$ is nonvanishing for $f \in U(r)$. Finally $\Phi[U(r)]$ is all of $V$ because the two domains have the same sections and $\Phi[U(r)]$ is full.

7. Other equivalence classes. The examples given here illustrate the variety of simply connected domains and their sections. I know of no overall classification scheme. One step beyond continuously tubular domains are those equivalent to some $U(r)$ where $r$ need be only lower semicontinuous. Lower semicontinuity insures that $U(r)$ is open. Call a domain tubular if it is equivalent to such a $U(r)$. The following theorem distinguishes equivalence classes of tubular domains. 
Theorem 7.1. Let $r, s: X \rightarrow(0, \infty]$ be lower semicontinuous. For $U(r)$ and $U(s)$ to be equivalent it is necessary and sufficient that $r$ and $s$ be infinite on the same set and there exist a nonvanishing fanction $a \in C(X)$ with $s(x) / r(x)=$ $a(x)$ when $r(x)<\infty$.

Proof. If the conditions are satisfied then $f \rightarrow$ af is an equivalence of $U(r)$ onto $U(s)$. Conversely let $\Phi$ be a given equivalence of $U(r)$ onto $U(s)$. The derivative $\Phi^{\prime}[0]$ is a continuous nonvanishing function; therefore $t=s /|\Phi[0]|$ is lower semicontinuous, and $t$ and $s$ are infinite on the same set. The mapping $\Psi=\Phi / \Phi^{\prime}[0]$ is an equivalence of $U(r)$ onto $U(t)$ and $\Psi^{\prime}[0]=1$. Put $g=\Psi[0] \epsilon$ $U(t)$. By Lemma 3.2 the section map $\Psi^{x}$ is an equivalence of $D(0, r(x))=$ $S_{x}(U(r))$ onto $D(0, t(x))=S_{x}(U(t))$. In particular $r$ and $t$ are infinite on the same set. When $r$ and $t$ are infinite the data $\Psi^{x}(0)=g(x)$ and $\Psi^{x^{\prime}}(0)=1$ imply $\Psi^{x}(z)=g(x)+z$. When $r$ and $t$ are finite, $\Psi^{x}$ is a fractional linear transformation. The data $\Psi^{x}(0)=g(x)$ and $\Psi^{x^{\prime}}(0)=1$ give us

$$
r(x)=t(x)-|g(x)|^{2} / t(x)
$$

and the expansion

$$
\Psi_{x}(z)=g(x)+z \sum_{n=0}^{\infty}(-\bar{g}(x))^{n}(r(x) t(x))^{-n} z^{n} .
$$

If we agree that $\bar{g} /(r t)$ vanishes when $r$ and $t$ are infinite, then the power series expansion of $\Psi$ at zero is $\Psi[f]=g+f \sum_{n=0}^{\infty}(-\bar{g} /(r t))^{n} f^{n}$. Since $\Psi$ is an analytic mapping in $C(X)$, the coefficients $(-\bar{g} /(r t))^{n}$ must be continuous. If $g=0$, then (7.1) forces $r=t$. In this case the nonvanishing function $a \in C(X)$ is $a=\left|\Phi^{\prime}[0]\right|$. In the general case $g /(r t)$ is some continuous function. The function $g$ is continuous, so $r t$ is continuous except possibly where $g$ vanishes. By (7.1) $t=\sqrt{ }\left(r t+|g|^{2}\right)$, and $t$ is continuous where $g$ is nonzero. For $r$ and $t$ finite (7.1) also gives

$$
f(x) / t(x)=1-|g(x)|^{2} /(t(x))^{2} .
$$

The positive lower semicontinuous function $t$ has a positive infimum on the compact space $X$. Equation (7.2) shows that as $x$ approaches the zero set of $\boldsymbol{g}$ or the set where $r$ and $t$ are infinite, the ratio $r(x) / t(x)$ approaches 1 . Put $b(x)=r(x) / t(x)$ where $g$ is nonzero and $r$ and $t$ are finite; otherwise put $b(x)=1$. The function $b$ is continuous. It is also positive, because $|g(x)|<t(x)$. The function $a=\left|\Phi^{\prime}[0]\right| / b$ is continuous and nonvanishing, and $s(x) / r(x)=a(x)$ when $s$ and $r$ are finite.

Example 1. A nontubular domain. Define $V \subset C([0,1])$ by

$$
V=\{f ;|f(x)|<2 \text { and }|f(1)-1|<1\} \text {. }
$$


Suppose $\Phi$ were an equivalence of some $U(r)$ onto $V$. Put $s=r\left|\Phi^{\prime}[0]\right|$ and $\Psi[f]=\Phi\left[f / \Phi^{\prime}[0]\right]$. The domain $U(s)$ is tubular, and $\Psi$ is an equivalence of $U(s)$ onto $V$ that satisfies $\Psi^{\prime}[0]=1$. Put $g=\Psi[0] \in V$.

The sections of $U(s)$ and $V$ are $s_{x}(U(s))=D(0, s(x)), s_{x}(V)=D(0,2)$ when $0 \leq x<1$ and $s_{1}(U(s))=D(0, s(1)), s_{1}(V)=D(1,1)$. By Lemma 3.2 the section map $\Psi^{x}$ is an equivalence of $S_{x}(U(s))$ onto $S_{x}(V)$. It follows that $\Psi^{x}$ is fractional linear, and its precise form can be calculated from the data $\Psi^{x}(0)=g(x)$ and $\Psi^{\prime}(0)=1$. For $0 \leq x<1$ we have

$$
\Psi^{x}(z)=g(x)+z\left\{1+z \bar{g}(x)\left(4-|g(x)|^{2}\right)^{-1}\right\}^{-1}
$$

Also

$$
\Psi^{1}(z)=g(1)+z\left\{1+z(\bar{g}(1)-1)\left(1-|1-g(1)|^{2}\right)^{-1}\right\}^{-1} .
$$

Expand $\Psi$ in a power series about zero. The coefficient functions must be continuous, in particular continuous at $x=1$. It turns out that $g(1)$ must satisfy

$$
g(1)\left(4-|g(1)|^{2}\right)^{-1}=(g(1)-1)\left(1-|1-g(1)|^{2}\right)^{-1} .
$$

By taking real and imaginary parts one sees that this equation has no solution; therefore $\Psi$ and hence $\Phi$ cannot exist.

The preceding example does have the property that all its sections are single sheeted. Call such a domain flat, and call a simply connected domain "flattenable" if it is equivalent to a flat domain. The next theorem shows that the sheets in the sections of a flattenable domain do not suddenly split apart or coalesce.

Theorem 7.2. Let $V$ be flattenable and let $g_{1}, g_{2}$ be functions in $V$. If $x(a) \rightarrow y \in X$ and $\sigma_{x(a)}\left(g_{1}\right)=\sigma_{x(a)}\left(g_{2}\right)$ for all $a$, then $\sigma_{y}\left(g_{1}\right)=\sigma_{y}\left(g_{2}\right)$. If $\sigma_{y}\left(g_{1}\right)=\sigma_{y}\left(g_{2}\right)$, then $\sigma_{x}\left(g_{1}\right) \in \Delta\left[x, g_{2}\right]$ for $x$ in some neigbborbood of $y$.

Proof. Let $U$ be a flat domain and $\Phi$ an equivalence of $U$ onto $V$. Determine $f_{1}, f_{2} \in U$ by $\Phi\left[f_{i}\right]=g_{i}$. Since $U$ is flat, $\sigma_{x}\left(f_{i}\right)=f_{i}(x)$. The section map relation (3.6) becomes $\Phi^{x}\left(f_{i}(x)\right)=\sigma_{x}\left(g_{i}\right)$.

Suppose $x(\alpha) \rightarrow y$ and $\sigma_{x(\alpha)}\left(g_{1}\right)=\sigma_{x(\alpha)}\left(g_{2}\right)$. Since $\Phi^{x(\alpha)}$ is one-to-one, we have $f_{1}(x(a))=f_{2}(x(a))$. The continuity of $f_{1}$ and $f_{2}$ implies that $f_{1}(y)=f_{2}(y)$. Thus

$$
\sigma_{y}\left(g_{1}\right)=\Phi^{y}\left(f_{1}(y)\right)=\Phi^{y}\left(f_{2}(y)\right)=\sigma_{y}\left(g_{2}\right) .
$$

Suppose $\sigma_{y}\left(g_{1}\right)=\sigma_{y}\left(g_{2}\right)$. This implies $f_{1}(y)=f_{2}(y)$. Choose $\epsilon>0$ so that $\Phi$ maps $B\left(f_{2}, \epsilon\right)$ into $B_{V}\left(\Phi\left[f_{2}\right]\right)=B_{V}\left(g_{2}\right)$. For all $x$ the section map $\Phi^{x}$ carries $D\left(f_{2}(x), \epsilon\right)=\sigma_{x}\left(B\left(f_{2}, \epsilon\right)\right)$ into $\Delta\left[x, g_{2}\right]=\sigma_{x}\left(B_{V}\left(g_{2}\right)\right)$. Choose a neighborhood $Y$ 
of $y$ on which $f_{1}(x)$ and $f_{2}(x)$ differ from $f_{1}(y)=f_{2}(y)$ by less than $\epsilon / 2$. For $x \in Y$ we have $f_{1}(x) \in D\left(f_{2}(x), \epsilon\right)$ and $\sigma_{x}\left(g_{1}\right)=\Phi^{x}\left(f_{1}(x)\right) \in \Delta\left[x, g_{2}\right]$.

Example 2. A domain whose section sheets suddenly split apart. In $C([0,4])$ let $b_{1}(x)=2+2 i, b_{2}(x)=12+2 i, b_{3}(x)=12+(12-2 x) i$ and $b_{4}(x)=(12-2 x) i$. Let $L$ be the path of functions $\left[b_{1}, b_{2}\right]+\left[b_{2}, b_{3}\right]+\left[b_{3}, b_{4}\right]$. Let $E$ be the planar set $\{x+i y ; 0 \leq x, y \leq 8\}$. Define $V$ in $C([0,4])$ by

$$
V=\{f ; f(x) \in E, 0 \leq x \leq 4\} \cup \bigcup_{f \in L} B(f, 2) .
$$

The section $S_{x}(V)$ is single sheeted for $0 \leq x \leq 1$, double sheeted for $1<x \leq 3$ and single sheeted for $3<x \leq 4$. See Figure 1 .
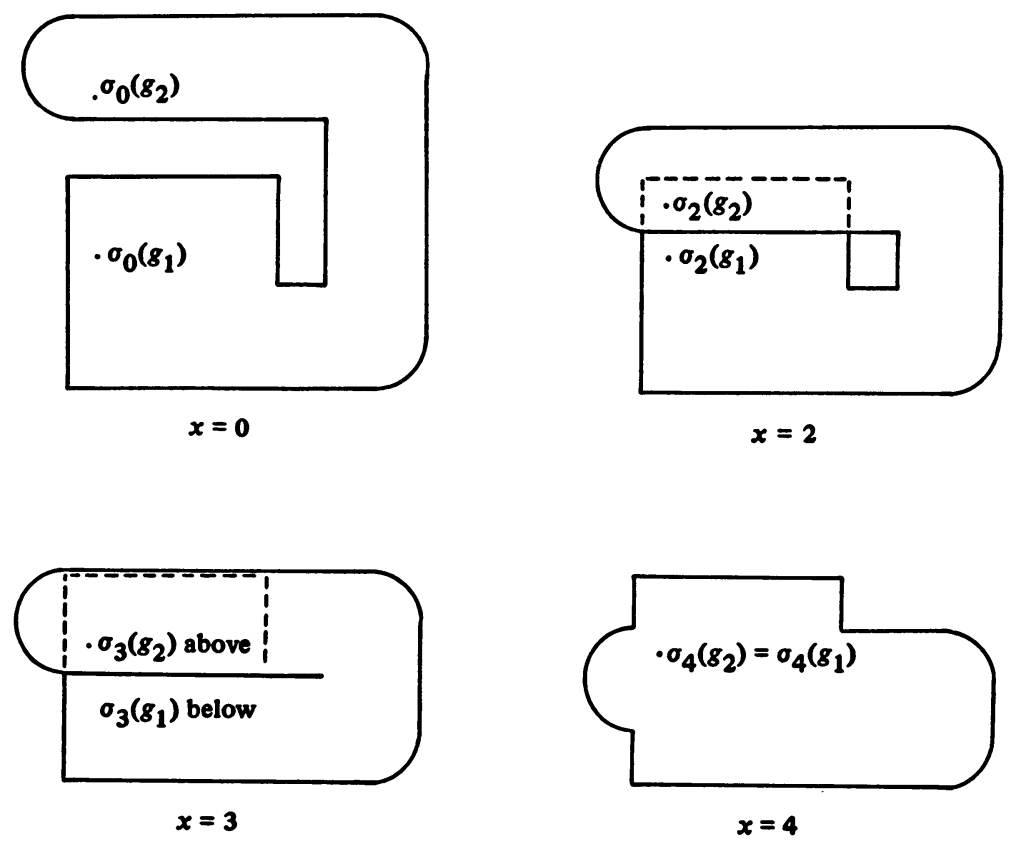

Figure 1

We define functions $g_{1}, g_{2} \in V$. Let $g_{1}$ have the constant value $1+5 i$. For $0 \leq x \leq 3$ put $g_{2}(x)=1+(11-2 x) i$, and for $3<x \leq 4$ put $g_{2}(x)=1+5 i$. For $3<x \leq 4$ we have $S_{x}(V)$ single sheeted and $\sigma_{x}\left(g_{1}\right)=\sigma_{x}\left(g_{2}\right)$. Yet $\sigma_{3}\left(g_{1}\right) \neq$ $\sigma_{3}\left(g_{2}\right)$. Notice that $g_{2} \in B_{V}\left(b_{4}\right)$. The first part of Theorem 7.2 shows that $V$ is not flattenable.

Example 3. A domain whose section sheets suddenly coalesce. The domain $V$ is to lie in $C([0,1])$. Rather than analytically define $V$ we refer to Figure 2. Regard the variable $t \in[0,1]$ as measuring time. Near time $t=0, S_{t}(V)$ is 

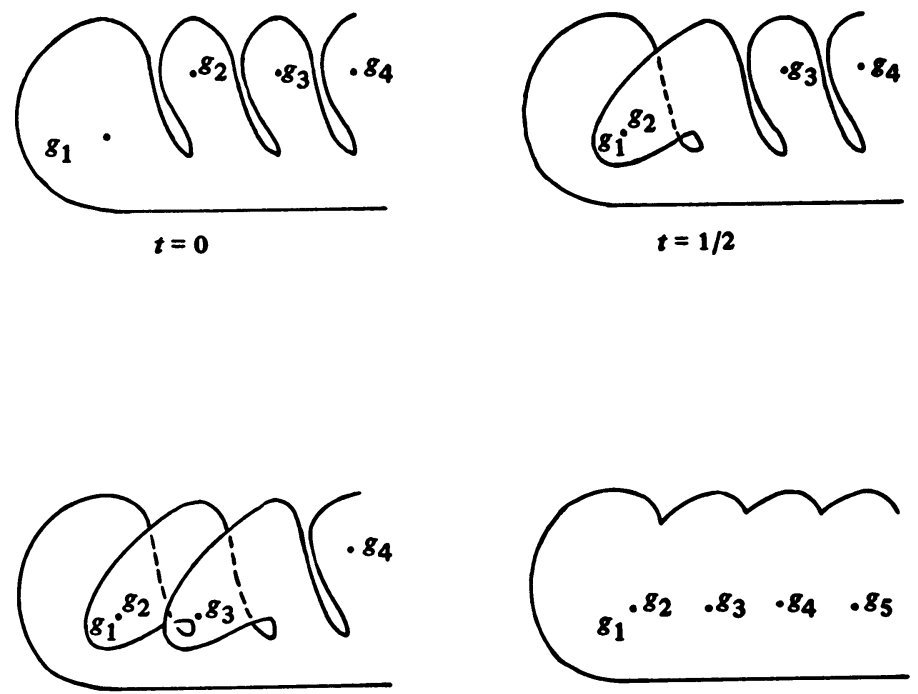

$t=3 / 4$

Figure 2

$t=1$

single sheeted. The values of functions $g_{1}, g_{2}$, etc. in $V$ are indicated by dots. The function $g_{1}$ is to be a constant. During $(0,1 / 2)$ the function value $g_{2}(t)$ bends over to $g_{1}$ and stays there for $t \geq 1 / 2$. However for $1 / 2 \leq t<1$ the section $S_{t}(V)$ is double sheeted over $g_{1}$, and $\sigma_{t}\left(g_{1}\right) \notin \Delta\left[t, g_{2}\right]$. During $(1 / 2,3 / 4)$ the function value $g_{3}(t)$ bends over; it and its neighbors cover the hole made by the $g_{2}$ arm. In general during $\left(1-2^{1-n}, 1-2^{-n}\right)$ the $g_{n+1}$ arm bends over and covers the hole made by the $g_{n}$ arm. At time $t=1$ all the holes are covered, and $S_{1}(V)$ is single sheeted. In particular $\sigma_{1}\left(g_{1}\right)=\sigma_{1}\left(g_{2}\right)$. The second part of Theorem 7.2 shows that $V$ is not flattenable.

It is instructive to follow through the construction of the section $S_{t}(V)$ when $t$ is slightly less than 1. If $t=1-2^{-n}$, then $\sigma_{t, j}\left(g_{1}\right)=\sigma_{t, j}\left(g_{2}\right)$ for $j<n$ and $\sigma_{t, n}\left(g_{1}\right) \neq \sigma_{t, n}\left(g_{2}\right)$. For this domain $V$ we have $S_{t}(V)=S_{t, \omega}(V)$ where $\omega$ is the first infinite ordinal. The idea of a sequence of overlapping arms can be extended to give examples with $S_{x} \neq S_{x, \omega}$.

\section{REFERENCES}

1. L. V. Ahlfors and L. Sario, Riemann surfaces, Princeton Math. Series, no. 26, Princeton Univ. Press, Princeton N. J., 1960, p. 180. MR 22 \#5729.

2. B. W. Glickfeld, On the inverse function theorem in commutative Banach algebras, Illinois J. Math. 15 (1971), 212-221. MR 42 \#8287.

3. G. M. Goluzin, Geometric theory of functions of a complex variable, GITTL, Moscow, 1952; English transl., Transl. Math Monographs, vol. 26, Amer. Math. Soc., Providence, R. I., 1969, p. 54. MR 15, 112; 40 \#308. 
4. M. Heins, Complex function theory, Pure and Appl. Math., vol. 28, Academic Press, New York, 1968, p. 343. MR 39 \#413.

5. E. Hille, Analytic function theory. Vol II, Introduction to Higher Math., Ginn, Boston, Mass., 1962, p. 236. MR 34 \#1490.

6. S.-T. Hu, Elements of general topology, Holden-Day, San Francisco, Calif., 1964, p. 71. MR 31 \#1643.

7. E. R. Lorch, The theory of analytic functions. in normed abelian vector rings, Trans. Amer. Math. Soc. 54 (1943), 414-425. MR 5, 100.

8. S. Saks and A. Zygmund, Analytic functions, 2nd ed., Monografie Mat., Tom 10, PWN, Warsaw, 1938; English transl., Monografie, Mat., Tom 28, PWN, Warsaw, 1965, p. 231. MR 31 \#4889.

9. H. E. Warren, A Riemann mapping theorem for $C(X)$, Proc. Amer. Math. Soc. 28 (1971), 147-154. MR 43 \#5300.

10. - Sets in $C(X)$ analytically equivalent to the open ball, Duke Matho J. 39 (1972), 711-717.

DEP ARTMENT OF MATHEMATICS, UNIVERSITY OF ARIZONA, TUCSON, ARIZONA 85721 